山्यRANÇAISE

$>\mathrm{DE}$

$\stackrel{1=1}{\simeq}$ PÉDAGOGIE

\section{Revue française de pédagogie}

Recherches en éducation

191 | avril-mai-juin 2015

Les descendants d'immigrés à l'école

\title{
Les descendants d'immigrés à l'école en France : entre discontinuité culturelle et discrimination systémique
}

Students of immigrant descent in France: between cultural discontinuity and systemic discrimination

Georges Felouzis, Barbara Fouquet-Chauprade et Samuel Charmillot

\section{OpenEdition \\ Journals}

Édition électronique

URL : http://journals.openedition.org/rfp/4738

DOI : $10.4000 /$ rfp.4738

ISSN : 2105-2913

Éditeur

ENS Éditions

Édition imprimée

Date de publication : 30 juin 2015

Pagination : 11-27

ISBN : 978-2-84788-772-3

ISSN : 0556-7807

\section{Référence électronique}

Georges Felouzis, Barbara Fouquet-Chauprade et Samuel Charmillot, «Les descendants d'immigrés à l'école en France : entre discontinuité culturelle et discrimination systémique », Revue française de pédagogie [En ligne], 191 | avril-mai-juin 2015, mis en ligne le 30 juin 2018, consulté le 04 janvier 2020. URL : http://journals.openedition.org/rfp/4738 ; DOI : 10.4000/rfp.4738 


\title{
Les descendants d'immigrés à l'école en France : entre discontinuité culturelle et discrimination systémique
}

\author{
Georges Felouzis \\ Barbara Fouquet-Chauprade \\ Samuel Charmillot
}

\begin{abstract}
Comment rendre compte des inégalités d'acquis scolaires liées aux parcours migratoires des élèves et de leur famille? Pour certains auteurs, ces inégalités seraient la conséquence de «discontinuités culturelles» entre les élèves de milieu populaire et/ou migrants et l'école elle-même. Ce type d'explication a longtemps été hégémonique en France. On peut cependant aussi les expliquer par des discriminations systémiques. À partir d'une analyse secondaire des données PISA 2003 et 2012 pour la France, cet article vise à mettre à l'épreuve des faits ces deux univers interprétatifs afin de rendre compte des inégalités scolaires liées au statut migratoire des élèves, l'hypothèse centrale étant qu'il existe un lien entre l'accentuation de la ségrégation scolaire des migrants d'une part et l'évolution des inégalités d'acquis de l'autre.
\end{abstract}

Mots-clés (TESE) : système éducatif, inégalité sociale, migration, discrimination.

L'évolution des inégalités entre élèves natifs et migrants en France constitue un terrain particulièrement fécond pour examiner les sources et les mécanismes de production des inégalités scolaires. Au-delà des débats particulièrement vifs qui ont agité la sociologie de l'éducation entre les tenants d'une interprétation « dispositionnaliste» (Bourdieu, 2013) et "stratégique» (Boudon, 1973) de ces inégalités, nous proposons dans cet article de reformuler la question des sources et mécanismes de production des inégalités scolaires. Notre questionnement porte sur la part respective de l'école et de la famille dans leur construction. Cette question prend aujourd'hui une ampleur nouvelle au regard de l'accroissement des inégalités d'apprentissage mesurées en France par des sources et des enquêtes très variées, utilisant des méthodes et des données qui ne le sont pas moins. Que l'on consulte les notes d'information de la DEPP (Rocher, 2008; 
Rocher \& Le Donné, 2012), les résultats des enquêtes internationales PISA (OCDE, 2013b; Felouzis \& Charmillot, 2012), ou encore des travaux plus localisés mêlant approches quantitatives et qualitatives (Broccolichi, Ben Ayed \& Trancart, 2010), le constat d'un accroissement des inégalités d'acquis entre élèves, et entre groupes d'élèves, s'impose comme une réalité qu'il appartient aux sciences sociales de comprendre et d'expliquer. Et ce d'autant plus que ces inégalités s'observent à tous les niveaux de scolarité, de l'entrée à l'école primaire à la sortie de l'enseignement secondaire, impliquant à la fois les inégalités qualifiées de «primaires »-au sens où elles dépendraient de la socialisation familiale - et celles qualifiées de "secondaires", c'est-à-dire liées au fonctionnement de l'école ellemême.

\section{Discontinuité culturelle versus discriminations systémiques : les termes du débat}

La littérature sur la question des sources et des mécanismes des inégalités scolaires est abondante. Dans un souci de synthèse, nous pouvons distinguer deux grands types d'explications qui se proposent de rendre compte de ces inégalités selon qu'elles privilégient les sources familiales ou institutionnelles comme facteurs premiers. II faut toutefois souligner que beaucoup d'auteurs empruntent à l'une et à l'autre de ces voies interprétatives, se différenciant par le dosage qu'ils proposent pour chacune d'elles.

La première explication privilégie l'idée de discontinuité culturelle entre la socialisation familiale d'une part et les attentes et normes scolaires de l'autre comme étant la source principale des inégalités d'expérience, de parcours et in fine d'apprentissage à l'école des élèves de groupes sociaux et/ou ethniques différents. Pour beaucoup d'auteurs en effet, notamment Lahire (2008), l'explication première s'inscrit - à partir d'une reformulation des travaux de Bourdieu et Passeron (1964) - en référence à cette idée de «discontinuité culturelle». Ce concept, issu de l'anthropologie de l'éducation américaine (van Zanten \& Anderson-Levitt, 1992), a d'abord été conçu pour rendre compte de l'échec scolaire massif des enfants des minorités à l'école. Dans le contexte français, il est utilisé indifféremment pour penser l'échec scolaire des que ces derniers sont «des enfants d'ouvriers comme les autres ». Dans cette perspective, l'entrée à l'école se fait pour certains élèves sur le mode de l'évidence, tant l'accord semble parfait entre les modèles familiaux valorisés et le monde scolaire. Pour d'autres, l'entrée à l'école équivaudrait à une sorte de rupture qui les plongerait dans l'étrangeté d'un monde nouveau et inconnu, comme Willis ([1978] 2011) a pu le montrer pour les élèves de la classe ouvrière anglaise. C'est ainsi que Lahire (2008) se demande si «l'inégale réussite scolaire des élèves issus des différents groupes sociaux ne met pas fondamentalement en jeu le rapport de ces groupes sociaux aux formes objectivées de culture et si les dispositions "méta" (le rapport scriptural-scolaire au langage), socialement constituées au sein de ces formes objectivées de culture, ne sont pas au centre des processus d'échec scolaire» (p.56).

Dans cet univers interprétatif, le handicap scolaire observé des élèves migrants ou directement issus de l'immigration serait à la fois le résultat de leur origine socioéconomique globalement plus défavorisée et de leur éloignement culturel lié à la migration. Au plan plus institutionnel, l'école est pensée ici comme « indifférente aux différences». En offrant le même enseignement à des élèves au départ inégaux, l'école produirait donc les inégalités d'acquis observées par les enquêtes nationales comme internationales.

Ce type d'explication a longtemps été hégémonique en France (voir par exemple Clerc \& Girard, 1964; Gratiot-Alphandéry \& Lambiotte, 1973; Vallet \& Caille, 1996). Pourtant, l'évolution récente et rapide de l'ampleur des inégalités scolaires liées notamment au statut migratoire laisse penser que d'autres facteurs sont à l'œuvre pour rendre compte de ces inégalités. Une première question pourrait notamment être de savoir s'il est certain que l'école reste « indifférente aux différences » et qu'elle offre à tous la même qualité d'éducation. L'hypothèse de l'indifférence aux différences se fonde essentiellement sur le postulat que l'école « républicaine » serait en accord avec les valeurs d'égalité des chances qu'elle proclame. Or, il est de bonne méthode d'examiner plus avant cette hypothèse, tant il est vrai que le fonctionnement réel des institutions sociales - dont l'école fait partie - est parfois fort éloigné de leur fonctionnement officiel. On peut par exemple voir dans les phénomènes ségrégatifs (Felouzis, 2003; Oberti, 2007) une forme d'inégalité de l'offre scolaire qui n'a rien à envier aux formes plus institutionnalisées de séparation précoce des élèves dans des filières différenciées et hiérarchisées (Felouzis, 
Charmillot \& Fouquet-Chauprade, 2011, 2013). Dans ce cadre, une part non négligeable des inégalités liées aux parcours migratoires et à l'origine sociale serait due aux phénomènes ségrégatifs et à leurs conséquences sur la qualité de l'enseignement (van Zanten, 2012). De même, les effets de composition liés à la ségrégation scolaire ont des conséquences avérées sur la progression des élèves (Dumay, Dupriez \& Maroy, 2010), voire sur des dimensions non académiques (Fouquet-Chauprade, 2013), même si les résultats de recherches varient d'un pays à l'autre et que les débats restent vifs pour savoir quelles sont la nature et l'ampleur de ces effets de composition (Duru-Bellat, 2002).

II s'agirait alors, selon le concept forgé par Merton (1968), d'un "effet Matthieu» (Crahay, 2013) dont le principe de base consiste à «donner plus à ceux qui ont le plus». Aux «meilleurs élèves», ceux des milieux sociaux les plus favorisés, on offre les enseignants les plus expérimentés, les meilleures conditions d'enseignement et d'apprentissage, les meilleures classes, etc. (Merle, 2012). On n'est donc plus ici dans le cadre d'une «indifférence aux différences» mais bien dans la production scolaire des inégalités par le biais de discriminations systémiques liées à un ensemble de facteurs urbains, sociaux et scolaires.

Le présent article ne prétend pas trancher de façon définitive entre ces deux modèles interprétatifs. Il propose plus modestement de les mettre à l'épreuve des faits à partir de l'analyse de l'évolution des inégalités liées aux parcours migratoires des élèves en France entre 2003 et 2012 à partir des données PISA.

Après avoir présenté ces données et leurs limites, nous montrerons que la théorie de la discontinuité culturelle n'est pas suffisante pour expliquer l'accroissement des inégalités de compétence liées au parcours migratoire des élèves et/ou de leur famille entre 2003 et 2012 , ce qui suggère que d'autres facteurs que ceux liés aux conditions familiales interviennent dans le renforcement des inégalités scolaires.

\section{Les données PISA comme source empirique}

L'usage des bases de données PISA présente certains avantages et inconvénients. L'enquête présente des avantages certains : échantillonnage représentatif des élèves de 15 ans, quelle que soit leur structure de scolarisation; mesure des acquis de ces élèves dans trois domaines-clés (compréhension de l'écrit, mathématiques, sciences); mesure précise des caractéristiques de leur milieu familial et scolaire; récurrence de l'enquête tous les 3 ans depuis 12 ans permettant des comparaisons diachroniques; ampleur internationale de l'enquête qui donne à voir l'évolution de chaque pays par rapport à tous les autres participant à l'enquête. Cependant, elle présente aussi des limites dues au fait qu'elle mesure des compétences et non des savoirs acquis en relation avec les programmes scolaires, ce qui limite sa capacité à rendre compte de la qualité de l'enseignement et plus généralement de l'école elle-même (Suchaut, 2015). On trouvera dans Felouzis et Charmillot (2012) une discussion approfondie des qualités et limites des enquêtes PISA, ainsi que des conditions d'usage de ces bases de données pour la recherche.

Dans le cadre de cet article, nous nous focalisons sur les élèves scolarisés en France ayant participé aux enquêtes PISA 2003 ( $N=4300$ ) et PISA $2012(N=4613)^{1}$. Le choix de comparer l'évolution des inégalités entre ces dates, plutôt qu'à partir de l'enquête PISA 2000, se justifie par le fait que lors de chaque cycle PISA un domaine de compétence est évalué plus en profondeur. En 2003 comme en 2012, l'accent a été mis sur l'évaluation des compétences en mathématiques. Très concrètement, cela signifie que les deux tiers du temps de test sont consacrés à évaluer ce domaine de compétence, tandis que la compréhension de l'écrit et les sciences font l'objet d'une évaluation plus succincte. Nos analyses porteront donc en priorité sur les mathématiques, même si nous ferons également référence aux deux autres domaines testés par PISA.

Le niveau de compétence en mathématiques, en compréhension de l'écrit et en sciences est estimé par un ensemble de "valeurs plausibles» qui permettent de prendre en compte le fait que les tests PISA sont constitués d'items ayant un niveau de difficulté variable et que tous les élèves n'ont pas répondu aux mêmes items. Pour les mathématiques, le niveau de compétence moyen dans les pays de l'OCDE est, par construction, de 500 points pour un écart-type de 100 en 2003, il est de 494 points pour un écart-type de 92 en 2012.

1 II s'agit ici des effectifs non pondérés. En 2003, I'enquête PISA a recueilli les réponses de 4300 élèves qui sont représentatifs des 734579 élèves de 15 ans scolarisés en France cette année-là. En 2012, les 4613 élèves de l'échantillon sont représentatifs de 699779 élèves. Pour plus de détails sur la méthode de pondération utilisée dans les enquêtes PISA, voir OCDE, 2005. 


\section{Les variables PISA utilisées dans cet article}

L'origine migratoire est mesurée par une variable qui distingue les élèves en fonction de leur lieu de naissance et celui de leurs parents. Les «natifs» représentent les élèves nés en France de parents nés en France, les « $2^{\mathrm{e}}$ génération » ceux qui sont nés en France de parents nés à l'étranger et les " $1^{\text {re }}$ génération " ceux qui sont nés à l'étranger de parents nés à l'étranger².

Le statut socioéconomique est mesuré par «l'indice de statut social, économique et culturel»(ESCS) ${ }^{3}$. Pour l'ensemble des pays de l'OCDE, la moyenne est fixée à 0 et l'écart-type à 1. Une valeur élevée sur cet indice indique un statut socioéconomique élevé (OCDE, 2005).

L'indice de statut socioéconomique, social et culturel est construit à partir de trois variables mesurant différentes dimensions de l'environnement familial :

- l'indice de statut social des parents, qui indique le statut professionnel le plus élevé des deux parents sur la base de «l'indice socioéconomique international de statut occupationnel» (ISEI) (Ganzeboom, De Graf \& Treiman, 1992);

- l'indice des possessions du foyer (HOMEPOS) est un indicateur du niveau de richesse de la famille. II prend en compte les possessions matérielles, culturelles et éducatives du foyer;

- l'indice du plus haut niveau d'étude des parents converti en années d'études (HISCED). Cette variable se base sur la Classification internationale type de l'éducation (CITE) élaborée par l'UNESCO, qui distingue sept niveaux d'éducation allant du pré-primaire (CITE 0) à l'enseignement supérieur de deuxième cycle (CITE 6) (UNESCO, 2006). Nous avons recodé cette variable en trois catégories : enseignement primaire (CITE 0-2), enseignement secondaire 2 (CITE 3-4), enseignement supérieur (CITE 5-6).

\section{De la discontinuité culturelle au plafond de verre : l'évolution des inégalités de compétence selon le statut migratoire}

Une première question est de savoir comment les caractéristiques des élèves natifs et non natifs ont évolué en France entre 2003 et 2012. On donne, pour cela, le tableau 1 qui propose une comparaison des deux enquêtes PISA.

La proportion d'élèves non natifs reste stable entre les deux dates ( $+0,7$ point de pourcentage), avec une légère baisse pour les $2^{\mathrm{e}}$ génération $(-0,8$ point de pourcentage), alors que la proportion de $1^{\text {re }}$ génération passe de 3,5\% à $5 \%$. L'âge moyen d'arrivée en France tend à légèrement baisser en passant de 6,3 à 6,1 ans de 2003 à 2012. Ces données reflètent dans ses grandes lignes les évolutions des migrations en France telles que les décrit l'INSEE ${ }^{4}$. Plus étonnante est la répartition

2 Faute de mieux, et par souci de synthèse, nous utiliserons la notion de «non-natifs» pour qualifier les élèves de $1^{\text {re }}$ et $2^{\mathrm{e}}$ génération.

3 Pour l'enquête 2012, le consortium PISA a changé l'algorithme permettant de calculer la variable ESCS. Il est par conséquent nécessaire d'utiliser une version «rééchelonnée » de la variable ESCS pour pouvoir comparer les données PISA 2003 à celles de PISA 2012. Pour plus de détails, voir OCDE, 2013a.

$4 \quad$ L'enquête Trajectoire et Origines montre par exemple que la France a la particularité de compter davantage de descendants d'immigrés que d'immigrés (Bouvier, 2012). En 2012, le recensement des élèves de 15 ans selon le sexe. II naît toujours plus de garçons que de filles pour une année donnée et l'équilibre démographique entre les deux sexes ne se fait qu'aux alentours de 25 ans au regard de la surmortalité masculine. À 15 ans, la population française est composée d'environ $51 \%$ de garçons et de $49 \%$ de filles (INSEE, 2014a). Or, l'échantillon PISA est représentatif des élèves de 15 ans et ceux-ci sont plus souvent des filles (52 \%) que des garçons $(48 \%)^{5}$. Ce phénomène s'accentue chez les non-natifs, notamment pour les $2^{\mathrm{e}}$ génération dans l'échantillon 2003 (58\% de filles), montrant le poids de la déscolarisation sur les destins scolaires des garçons issus de l'immigration (Bernard, 2011) ainsi que leur orientation plus fréquente vers les classes spécialisées (Vallet \& Caille, 1996) exclues des échantillons PISA.

Au plan du statut socioéconomique, les élèves natifs et non natifs tendent à se rapprocher entre 2003 et 2012 : les $2^{\mathrm{e}}$ génération sont moins défavorisés par rapport aux natifs en 2012 qu'en 2003, et il en est de même pour les $1^{\text {re }}$ génération. Une lecture plus précise en fonction des différentes dimensions de l'indice de statut socioéconomique montre que la situation des élèves de $1^{\text {re }}$ génération s'est améliorée au plan de leur capital culturel, mesuré par le niveau d'études des

de la population relevait que la France comptait $8 \%$ de descendants d'immigrés (7,5 \% en 1999) et $5 \%$ d'étrangers nés à l'étranger $(4,5 \%$ en 1999).

5 Ce phénomène se retrouve dans les données de la DEPP et reflète la déscolarisation plus fréquente des garçons que des filles avant la fin de la scolarité obligatoire. 
Tableau 1. Évolution des caractéristiques des élèves natifs, de $1^{\text {re }}$ et de $2^{\mathrm{e}}$ génération entre 2003 et 2012

\begin{tabular}{|c|c|c|c|c|c|c|}
\hline & \multicolumn{2}{|c|}{ Natifs } & \multicolumn{2}{|c|}{$2^{\mathrm{e}}$ génération } & \multicolumn{2}{|c|}{$1^{\text {re }}$ génération } \\
\hline & 2003 & 2012 & 2003 & 2012 & 2003 & 2012 \\
\hline$\%$ dans la population totale & 85,7 & 85,0 & 10,8 & 10,0 & 3,5 & 5,0 \\
\hline$\%$ de filles & 52,2 & 51,7 & 57,9 & 53,1 & 51,0 & 51,8 \\
\hline \multicolumn{7}{|l|}{ Langue parlée } \\
\hline Français & 98,7 & 97,3 & 62,7 & 68,5 & 47,3 & 41,5 \\
\hline Langue régionale & 0,5 & 1,2 & 1,6 & 1,2 & 0,0 & 5,3 \\
\hline Autre langue & 0,8 & 1,5 & 35,7 & 30,2 & 52,7 & 53,2 \\
\hline Âge d'arrivée en France & & & & & 6,3 & 6,1 \\
\hline$\%$ de familles monoparentales & 19,5 & 14,1 & 20,1 & 14,1 & 20,9 & 14,2 \\
\hline$\%$ de redoublants & 38,4 & 25,4 & 39,9 & 41,0 & 42,9 & 45,8 \\
\hline $\begin{array}{l}\text { Indice de statut socioécono- } \\
\text { mique (ESCS) }\end{array}$ & 0,12 & 0,11 & $-0,71$ & $-0,61$ & $-0,70$ & $-0,60$ \\
\hline $\begin{array}{l}\text { Indice statut social des parents } \\
\text { (ISEI) }\end{array}$ & 0,07 & 0,07 & $-0,54$ & $-0,46$ & $-0,28$ & $-0,44$ \\
\hline $\begin{array}{l}\text { Indice des possessions du foyer } \\
\text { (HOMEPOS) }\end{array}$ & 0,09 & 0,08 & $-0,45$ & $-0,33$ & $-0,64$ & $-0,59$ \\
\hline \multicolumn{7}{|l|}{$\begin{array}{l}\text { Plus haut niveau d'étude des } \\
\text { parents (HISCED) }\end{array}$} \\
\hline \% d'enseignement obligatoire & 13,1 & 6,7 & 46,5 & 26,1 & 44,9 & 26,2 \\
\hline $\begin{array}{l}\% \text { d'enseignement } \\
\text { secondaire } 2\end{array}$ & 47,6 & 36,5 & 30,9 & 39,4 & 23,0 & 26,3 \\
\hline$\%$ d'enseignement supérieur & 39,4 & 56,8 & 22,6 & 34,6 & 32,1 & 47,5 \\
\hline
\end{tabular}

Note : les variables ESCS, ISEl et HOMEPOS sont standardisées sur la moyenne de la France (moyenne 0, écart-type 1).

parents : entre 2003 et 2012, le pourcentage d'élèves de $1^{\text {re }}$ génération ayant des parents diplômés de l'enseignement supérieur est passé de $32,1 \%$ à $47,5 \%{ }^{6}$. Leur situation s'est en revanche dégradée au plan de leur statut socioprofessionnel (-0,28 en 2003 contre -0,44 en 2012 pour l'indice de statut social des parents).

En définitive, qu'observe-t-on au plan des caractéristiques des élèves natifs et non natifs en France? D'abord une stabilité de ces caractéristiques et une amélioration globale du statut socioéconomique des $2^{\mathrm{e}}$ génération. Les évolutions sont plus nuancées pour les $1^{\text {re }}$ génération : ils sont plus nombreux en 2012 qu'en 2003 , ont des parents plus diplômés mais dont le statut socioprofessionnel s'est dégradé.

6 Ces chiffres vont dans le même sens que les données de l'INSEE (2014b) qui indiquent qu'entre 2004 et 2012, le pourcentage d'immigrés ayant un diplôme au moins équivalent au baccalauréat a augmenté de 7 points.
Compte tenu de ces premiers résultats, et si l'on se situe dans la perspective de la théorie de la discontinuité culturelle, on ne devrait pas observer de changements profonds au plan des inégalités de compétence entre élèves en fonction de leur statut migratoire. On devrait même observer une amélioration de leurs résultats tant il est vrai qu'au plan du statut socioéconomique et du niveau d'études de leurs parents, la distance sociale entre ces élèves et l'école tend plutôt à se réduire. Une première comparaison des résultats des élèves entre les deux enquêtes PISA (tableau 2) apporte des éléments de réponse. Le tableau 2 montre tout d'abord la baisse des scores des élèves en mathématiques et en sciences. Pour la compréhension de l'écrit, la comparaison 20032012 indique une augmentation des scores. II faut dire que les scores moyens en compréhension de l'écrit avaient baissé entre 2000 et 2009 passant de 505 en moyenne à 496. Le niveau moyen en 2012 est donc revenu à son niveau initial (505 en moyenne). 
Tableau 2. Évolution des scores aux tests PISA entre 2003 et 2012 en fonction du statut migratoire

\begin{tabular}{|c|c|c|c|c|c|c|c|c|c|c|c|c|}
\hline & \multicolumn{3}{|c|}{ Natifs } & \multicolumn{3}{|c|}{$2^{\mathrm{e}}$ génération } & \multicolumn{3}{|c|}{$1^{\text {re }}$ génération } & \multicolumn{3}{|c|}{ Évolution Diff. Score 2003-2012 } \\
\hline & 2003 & 2012 & Évolution & 2003 & 2012 & Évolution & 2003 & 2012 & Évolution & $\begin{array}{l}\text { Natifs } \\
2^{e} \text { gén. }\end{array}$ & $\begin{array}{l}\text { Natifs } \\
1^{\text {re }} \text { gén }\end{array}$ & $\begin{array}{l}1^{\text {re }} \text { gén. } \\
2^{\text {e }} \text { gén. }\end{array}$ \\
\hline Mathématiques & 521 & 508 & -13 & 473 & 449 & -24 & 445 & 425 & -20 & $11^{*}$ & 7 & 4 \\
\hline Écrit & 506 & 519 & 13 & 456 & 464 & 8 & 424 & 423 & -1 & 5 & 14 & -9 \\
\hline Sciences & 523 & 513 & -10 & 465 & 442 & -23 & 429 & 422 & -7 & 13 & -3 & 16 \\
\hline
\end{tabular}

Lecture : * : 'écart de score en mathématiques entre les élèves natifs et de 2e génération augmente de 11 points entre 2003 et 2012.

L'apport de ce tableau est plus de rendre compte de l'évolution des inégalités liées aux parcours migratoires que des scores bruts en eux-mêmes. De façon générale, lorsque la tendance est à la baisse entre les deux dates, cette tendance s'avère plus marquée pour les élèves de $1^{\text {re }}$ et de $2^{\mathrm{e}}$ génération que pour les natifs. Ce phénomène s'observe pour les mathématiques : alors que les natifs perdent en moyenne 13 points entre 2003 et 2012, les élèves de $2^{\mathrm{e}}$ génération en perdent 24 et les $1^{\text {re }}$ génération 20 . II en est de même en sciences pour les élèves de $2^{\mathrm{e}}$ génération : ils perdent 23 points alors que les natifs n'en perdent que 10 dans la même période (les $1^{\text {re }}$ génération perdent pour leur part 7 points).

Qu'en est-il pour la compréhension de l'écrit? Entre les deux dates, le score de l'ensemble des élèves est orienté à la hausse (de 496 en 2003 à 505 en 2012), mais cette hausse est plus marquée pour les natifs $(+13)$ que pour les $2^{\mathrm{e}}$ génération $(+8)$ et pour les $1^{\text {re }}$ génération dont le score reste stable $(-1)$. Ces évolutions contrastées vont dans le sens d'une accentuation progressive du fossé entre natifs et non-natifs. C'est ce que montrent les trois dernières colonnes du tableau 2 : l'écart se creuse entre les élèves natifs et de $2^{\mathrm{e}}$ génération dans les trois domaines de compétence. Le phénomène est comparable entre les natifs et les élèves de $1^{\text {re }}$ génération, sauf en sciences où l'écart reste constant.

Ainsi, les caractéristiques des élèves non natifs restent globalement stables, voire s'améliorent au plan socioéconomique (tableau 1) alors que les écarts de compétence par rapport aux natifs tendent à augmenter (tableau 2). Ces premiers constats méritent d'être approfondis car ils suggèrent des mécanismes différenciés de production des inégalités scolaires, comme si l'origine socioéconomique des élèves jouait un rôle différent pour les natifs et les non-natifs. On aurait dû la discontinuité culturelle, une amélioration des scores relatifs des élèves de $2^{\mathrm{e}}$ et $1^{\text {re }}$ génération, mais ce n'est pas ce que donnent à voir les résultats. Pour explorer cette hypothèse, nous avons mis en œuvre des analyses de régression multivariées sur les données PISA 2003 et 2012 en mobilisant des variables de contrôle (sexe, statut migratoire, langue parlée à la maison, structure familiale, redoublement et milieu socioéconomique) dans la perspective de tester la nature et l'ampleur de l'interaction entre l'indice de statut socioéconomique et le statut migratoire?.

L'objectif de cette analyse de régression, dont les résultats sont consignés au tableau 3 , est donc de déterminer si, toutes choses étant égales par ailleurs ${ }^{8}$, l'effet de l'origine socioéconomique des élèves sur le score en mathématiques varie pour les natifs, les $1^{\text {re }}$ ou les $2^{\mathrm{e}}$ génération.

Une première lecture du tableau 3 confirme le renforcement des inégalités scolaires en France entre les deux dates considérées. La taille de l'effet des différentes variables augmente de façon notable entre 2003 et 2012. L'effet très négatif du redoublement s'accentue entre les deux enquêtes. Il passe de $-89,9$ à $-98,2$ points sur l'échelle de compétence en mathématiques. C'est aussi le cas pour le statut migratoire :

7 Le tableau en annexe décrit plus en détail les caractéristiques des élèves, en lien avec leur statut socioéconomique et leur statut migratoire.

8 Les analyses de régression multivariées permettent de prendre en compte les intercorrélations entre les différentes variables explicatives et ainsi d'isoler l'effet «net » de chaque variable. Il est ainsi possible de mettre en œuvre un raisonnement «toutes choses égales par ailleurs». Ce raisonnement comporte toutefois deux limites importantes dont il faut tenir compte. Premièrement, lorsque l'on raisonne «toutes choses égales par ailleurs», les «choses égales par ailleurs ne sont autres que les variables incluses dans le modèle» (Bressoux, 2008, p. 98). La deuxième limite a trait à l'interprétation des analyses de régression et au risque de produire des résultats décontextualisés de la réalité, n'ayant plus aucune pertinence sociologique (Halbwachs, [1935] 1944; Vallet, 2002). 
Tableau 3. Effets des caractéristiques des élèves sur les scores en mathématiques en 2003 et 2012. Analyse de régression multivariée avec effet d'interaction entre le statut migratoire et le statut socioéconomique

\begin{tabular}{|c|c|c|}
\hline & PISA 2003 & PISA 2012 \\
\hline R2 du modèle & 42,4 & 43,3 \\
\hline Constante & $562,9 * * *$ & $539,0 * * *$ \\
\hline \multicolumn{3}{|l|}{ Sexe } \\
\hline Filles & $-19,4^{* * *}$ & $-16,2^{* * *}$ \\
\hline Garçons & réf. & réf. \\
\hline \multicolumn{3}{|l|}{ Statut migratoire IMMIG } \\
\hline $1^{\text {re }}$ génération & $-33,1^{* * *}$ & $-41,2^{* * *}$ \\
\hline $2^{\mathrm{e}}$ génération & $-22,8^{* * *}$ & $-26,6^{* * *}$ \\
\hline Natifs & réf. & réf. \\
\hline \multicolumn{3}{|l|}{ Langue parlée à la maison } \\
\hline Autre langue & $-4,1 * * *$ & $-9,2^{* * *}$ \\
\hline Autre dialecte national & $-10,3 * * *$ & $-17,6^{* * *}$ \\
\hline Langue du test & réf. & réf. \\
\hline \multicolumn{3}{|l|}{ Structure familiale } \\
\hline Famille monoparentale & n.s. & $1,3^{* * *}$ \\
\hline Famille avec deux parents ou autre & réf. & réf. \\
\hline \multicolumn{3}{|l|}{ Redoublement } \\
\hline En retard & $-89,9 * * *$ & $-98,2^{* * *}$ \\
\hline À l'heure & réf. & réf. \\
\hline $\begin{array}{l}\text { Indice de statut socioéconomique } \\
\text { (standardisé) (ZESCS) }\end{array}$ & $24,8^{* * *}$ & $31,5^{* * *}$ \\
\hline \multicolumn{3}{|l|}{ Interaction IMMIG * ZESCS } \\
\hline $1^{\text {re }}$ génération * ZESCS & $24,2^{* * *}$ & $-8,3 * * *$ \\
\hline $2^{\mathrm{e}}$ génération ${ }^{*} \mathrm{ZESCS}$ & $-1,7^{* * *}$ & $-17,4^{* * *}$ \\
\hline Natifs * ZESCS & réf. & réf. \\
\hline
\end{tabular}

Note : ${ }^{* *}$ : significatif au seuil de 0,001 .

Lecture : en 2003, le score en mathématiques des filles est de 19,4 points inférieur à celui des garçons (réf.). Le score des $1^{\text {re }}$ génération $\left(-33,1\right.$ points) et des $2^{e}$ génération (-22,8 points) est inférieur à celui des natifs (réf.). Les élèves en retard scolaire obtiennent 89,9 points de moins que ceux à l'heure (réf.). À chaque fois que l'indice de statut socioéconomique augmente d'un point, le score en mathématiques augmente de 24,8 points. 
Figures 1 et 2 . Score en mathématiques prédit en fonction du statut socioéconomique et du statut migratoire

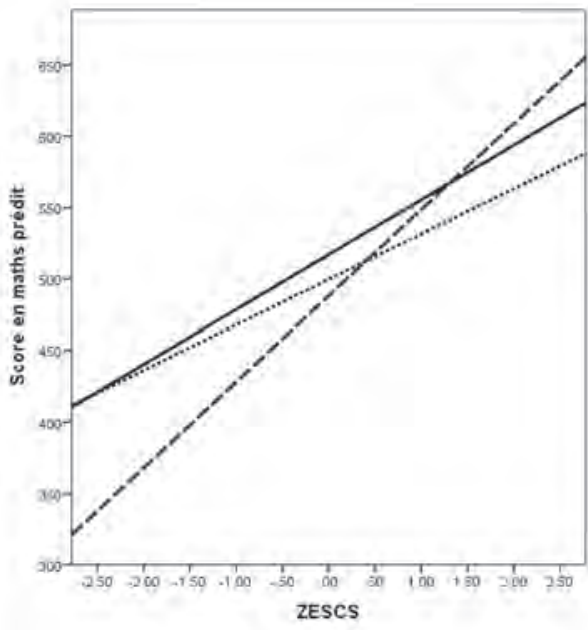

Figure 1. PISA 2003

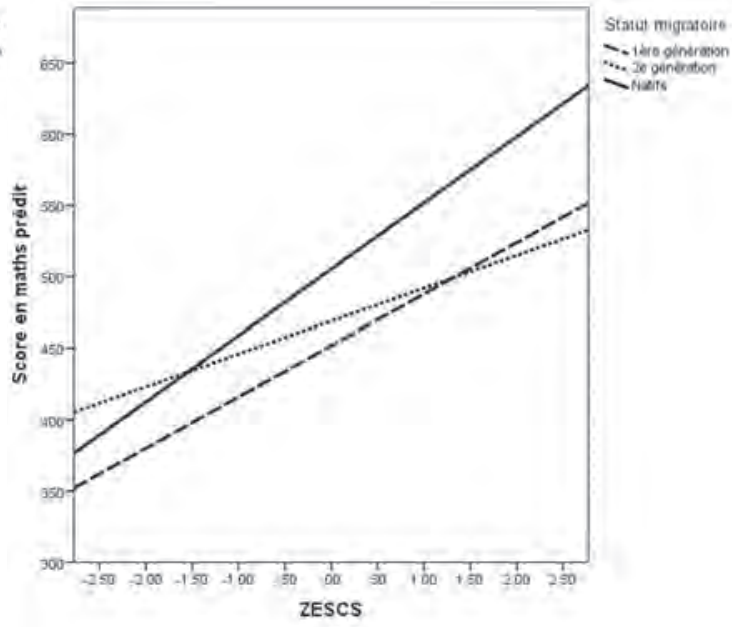

Figure 2. PISA 2012 comparés aux élèves natifs, les $2^{\mathrm{e}}$ génération ont - 26,6 points en 2012 contre $-22,8$ points en 2003. Pour les $1^{\text {re }}$ génération, l'évolution est de même ampleur : -41,2 points en 2012 contre -33,1 points en 2003. Compte tenu du fait que nous mesurons ici des effets nets, on ne peut invoquer une transformation de la population migrante ou issue de l'immigration pour expliquer ce renforcement des inégalités, même s'il n'est pas exclu que des variables non incluses dans l'analyse puissent rendre compte d'une telle évolution.

Les autres variables montrent la même tendance : un renforcement du lien entre caractéristiques sociales des élèves et leurs scores. En 2012, un point de plus sur I'indice de statut socioéconomique implique 31,5 points de plus en mathématiques, contre 24,8 points en 2003. L'accroissement des inégalités scolaires en France ne concerne donc pas seulement les élèves non natifs. Les élèves défavorisés par leur milieu socioéconomique notamment voient eux aussi leurs acquis nettement baisser par rapport aux plus favorisés (Felouzis, 2014).

L'interaction entre le statut socioéconomique des élèves et leur statut migratoire est très significative ( $p=0,000$ ) en 2003 comme en 2012, ce qui signifie que l'indice de statut socioéconomique n'a pas le même effet sur les scores pour les élèves natifs d'une part et non natifs de l'autre. Ajoutons que la taille de l'effet de cette interaction sur les scores varie entre les deux ration est de $-17,4$ points. Pour lire ce coefficient, il faut se reporter à l'effet net de l'ESCS indiqué au tableau 3. En 2012, un point de plus sur cet indice implique 31,5 points de plus sur l'échelle de compétence en mathématiques. Mais dans le cas des élèves de $2^{\mathrm{e}}$ génération, le gain est inférieur de 17,4 points, ce qui nous donne $31,5-17,4=14,1$ points. Pour les $1^{\text {re }}$ génération, et selon le même calcul, on obtient 31,5-8,3 = 23,2 points. Nous vérifions ainsi notre hypothèse selon laquelle le niveau socioéconomique n'agit pas avec la même intensité pour les élèves natifs d'une part et non natifs de l'autre.

De façon plus précise, entre 2003 et 2012, la taille de cet effet d'interaction s'est accrue, notamment pour les élèves de $2^{\mathrm{e}}$ génération pour lesquels l'effet $d$ 'interaction passe de $-1,7$ points à $-17,4$ points. Pour les $1^{\text {re }}$ génération, il est positif en $2003(+24,2)$ et devient négatif en $2012(-8,3)$. Cela signifie qu'en 2012, les élèves non natifs tendent à être moins performants aux tests PISA, quel que soit leur statut socioéconomique et que ce phénomène est bien plus marqué pour les élèves de $2^{\mathrm{e}}$ génération. D'où une variance des pentes plus marquée en 2012 qu'en 2003, comme l'indiquent les figures 1 et 2 qui donnent le score en mathématiques prédit par l'analyse de régression multivariée présentée au tableau 3.

La comparaison de ces deux figures permet de mettre en évidence des évolutions différentes pour les natifs et les non-natifs : 
Tableau 4. Effets des caractéristiques des élèves sur les scores en mathématiques en 2003 et 2012. Analyse de régression multivariée avec effet d'interaction entre le statut migratoire et le niveau d'études des parents

\begin{tabular}{l|r|c}
\cline { 2 - 3 } & PISA 2003 & PISA 2012 \\
\hline Constante & $579.6^{* * *}$ & $555.8^{* * *}$ \\
\hline Variables de contrôle* & Oui & Oui \\
\hline Interaction entre le statut migratoire & $-112,3^{* * *}$ & $-74,0^{* * *}$ \\
et niveau d'études des parents (IMMIG*HISCED) & $-68,3^{* * *}$ & $-68,8^{* * *}$ \\
\hline $1^{\text {re }}$ gén. ${ }^{*}$ Ens. obligatoire & $-16,7^{* * *}$ & $-66,5^{* * *}$ \\
$1^{\text {re }}$ gén. ${ }^{*}$ Ens. secondaire 2 & $-63,6^{* * *}$ & $-62,8^{* * *}$ \\
$1^{\text {re }}$ gén. ${ }^{*}$ Ens. supérieur & $-38,9^{* * *}$ & $-51,5^{* * *}$ \\
$2^{\mathrm{e}}$ gén. ${ }^{*}$ Ens. obligatoire & $-32,8^{* * *}$ & $-43,6^{* * *}$ \\
$2^{\mathrm{e}}$ gén. ${ }^{*}$ Ens. secondaire 2 & $-27,6^{* * *}$ & $-57,9^{* * *}$ \\
$2^{\mathrm{e}}$ gén. ${ }^{*}$ Ens. supérieur & $-14,4^{* * *}$ & $-29,4^{* * *}$ \\
Natifs ${ }^{*}$ Ens. obligatoire & réf. & réf. \\
Natifs * Ens. secondaire 2 &
\end{tabular}

Note : * les variables de contrôle mobilisées dans le modèle sont les suivantes : sexe, langue parlée à la maison, structure familiale, redoublement; *** : significatif au seuil de 0,001 .

- pour les $1^{\text {re }}$ et $2^{\mathrm{e}}$ génération, la pente est plus faible en 2012 qu'en 2003, ce qui suggère que pour les élèves non natifs les inégalités sociales de compétence se sont réduites. Il ressort cependant que ce phénomène est surtout lié à une forte baisse du score des élèves non natifs les plus favorisés : alors qu'en 2003 ceux-ci réussissaient aussi bien voir mieux que les natifs, en 2012 leur score en mathématiques est beaucoup plus faible;

- pour les élèves natifs, l'évolution est inverse : la pente est plus forte en 2012 qu'en 2003. Les inégalités sociales de compétence se sont donc accentuées : les natifs les plus favorisés voient leur score augmenter légèrement, mais surtout le score des natifs les plus défavorisés baisse fortement.

Ces évolutions contrastées font qu'au final on assiste à une accentuation des inégalités entre natifs et nonnatifs. En 2003, parmi les élèves les plus défavorisés, les élèves natifs et de $2^{\mathrm{e}}$ génération se situent au même niveau d'acquis en mathématiques. Seuls les élèves de $1^{\text {re }}$ génération ont des scores plus faibles. Toutefois, plus le statut socioéconomique des élèves s'accroît, moins les différences entre élèves natifs et non natifs sont fortes, montrant la prédominance du facteur socioéconomique sur le facteur migratoire pour expliquer les inégalités scolaires. En 2012, la situation a radicalement changé. La distance s'est accrue entre élèves natifs et non natifs, notamment pour les plus favorisés, comme si la frontière migratoire s'était renforcée pour limiter les acquis scolaires des élèves de $1^{\text {re }}$ et $2^{\mathrm{e}}$ génération.

Là encore, on peut se questionner sur les sources de ce phénomène qui suggère que l'accroissement des inégalités de compétence entre les deux enquêtes PISA liées au statut migratoire ne serait pas dû à un accroissement de la distance culturelle entre l'école et les familles en tant que telle, mais à d'inégales opportunités d'apprentissage.

Pour approfondir cette analyse et identifier les sources de ce phénomène, il est utile de préciser, au sein même de l'indice de statut socioéconomique, le rôle du capital culturel dans ce processus. Comme indiqué dans l'encadré méthodologique, l'indice de statut socioéconomique rassemble des informations sur le capital culturel hérité (niveau d'études le plus élevé des deux parents), le statut social (le statut professionnel le plus élevé des deux parents) et le niveau de richesse de la famille (les possessions du foyer). Plus spécifiquement, si les plus faibles acquis scolaires des élèves non natifs étaient le résultat d'une distance culturelle accrue aux normes et implicites scolaires, nous devrions observer un effet significatif de l'interaction 
Figures 3 et 4 . Score en mathématiques prédit, en fonction du statut migratoire et du niveau d'études des parents

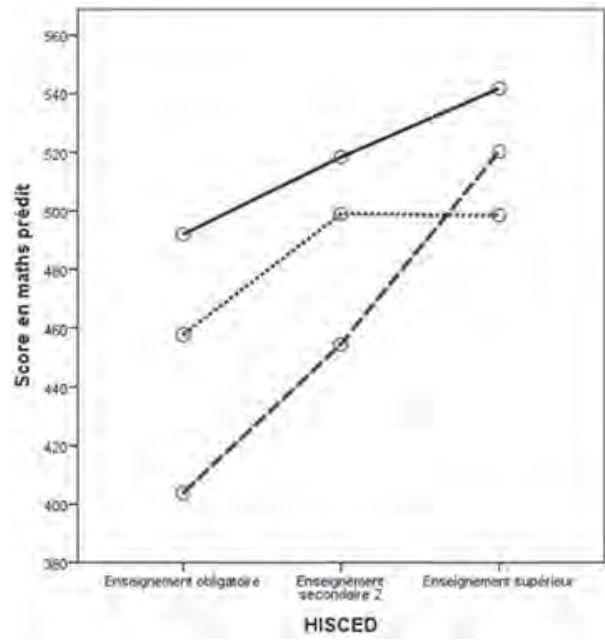

Figure 3. PISA 2003
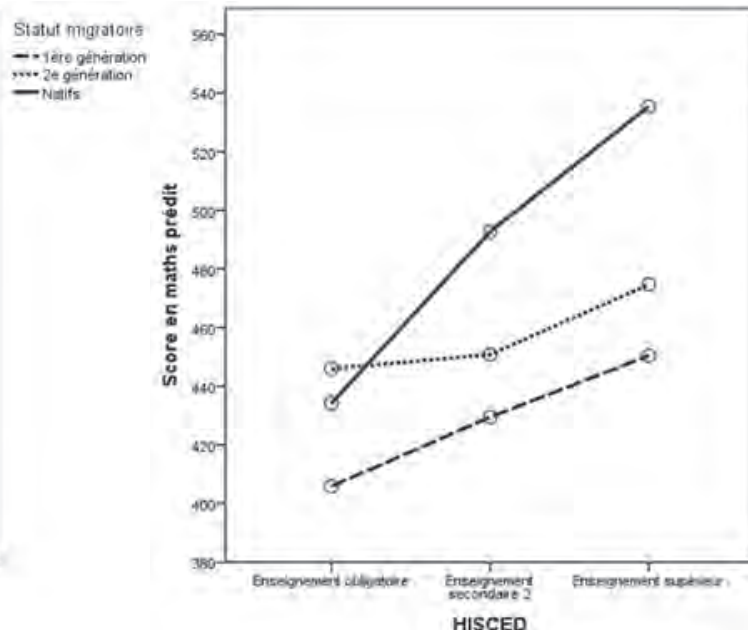

Figure 4. PISA 2012 entre le statut migratoire et le capital culturel. Effet qui devrait montrer que lorsque cette distance à l'école diminue en relation avec le capital culturel de la famille, les élèves non natifs acquièrent à l'école les mêmes compétences que les natifs.

La structure des inégalités de compétence s'est profondément transformée entre 2003 et 2012. L'interaction entre le statut migratoire et le niveau d'études le plus élevé des deux parents montre des évolutions notables entre les deux dates (voir tableau 4). Trois faits essentiels peuvent être soulignés :

- être migrant de $1^{\text {re }}$ génération et de parents ayant seulement atteint l'enseignement primaire est moins pénalisant en 2012 (-74) qu'en 2003 (-112,3). C'est le seul cas de figure pour lequel la taille de l'effet diminue entre les deux dates;

- pour les autres niveaux d'études des parents, le contraste s'accentue fortement pour les élèves non natifs comparés aux natifs : sur l'échelle de compétence en mathématiques, 16,7 points séparaient en 2003 les élèves de $1^{\text {re }}$ génération dont les parents ont atteint l'enseignement supérieur des élèves natifs de même capital culturel. En 2012, l'écart est de 66,5 points. Le même phénomène s'observe pour les $2^{\mathrm{e}}$ génération, mais de façon moins marquée : pour ceux dont les parents ont atteint l'enseignement supérieur, l'écart était de 32,8 points en 2003 , il est de 43,6 en 2012 ;

- l'écart se creuse aussi pour les élèves dont les parents ont atteint l'enseignement secondaire 2 : il reste stable pour les élèves de $1^{\text {re }}$ génération, mais s'accentue pour les $2^{\mathrm{e}}$ génération (-38,9 en $2003 ;-51,5$ en 2012) ainsi que pour les élèves natifs (-14,4 en 2003; -29,4 en 2012).

Ces résultats montrent des transformations profondes dans la structure même des inégalités, mêlant une dégradation notable des compétences des élèves natifs dont le capital culturel est très faible et des élèves non-natifs dont les parents ont effectué des études au niveau du secondaire 2 ou du supérieur. Pour mieux visualiser le sens de ces évolutions, on peut se reporter aux figures 3 et 4 - issues du tableau 4 - qui donnent les simulations des scores des élèves natifs, de $1^{\text {re }}$ et $2^{\mathrm{e}}$ génération selon le niveau d'études de leurs parents.

En 2003, l'effet du capital culturel est similaire quel que soit le statut migratoire des élèves. À quelques nuances près, nous avons là une illustration empirique du modèle de la discontinuité culturelle entre les élèves et l'école. Les élèves migrants ou issus de l'immigration ont plus souvent des parents avec un faible niveau d'études. Mais lorsque leur capital culturel est important, ils réussissent à l'école de façon comparable aux élèves natifs, même si leurs scores restent inférieurs à ceux des natifs de même condition.

En 2012, la situation est radicalement différente car le capital culturel hérité a beaucoup moins d'effet sur les scores des élèves de $1^{\text {re }}$ et de $2^{\mathrm{e}}$ génération. Quel que soit le niveau d'études de leurs parents, leurs scores plafonnent à un niveau très bas, alors que les acquis des natifs augmentent fortement selon cette variable. 
Cela suggère que le seul fait d'être non natif a des effets propres sur les opportunités d'apprentissage.

Ainsi, le handicap scolaire des élèves non natifs s'explique moins aujourd'hui qu'hier par leur origine sociale ou leurs conditions de vie, et beaucoup plus par le seul fait d'être non natifs de $1^{\text {re }}$ et surtout de $2^{\mathrm{e}}$ génération.

Ajoutons que la dégradation des scores est particulièrement marquée aussi pour les élèves de capital culturel faible qu'ils soient natifs ou non, suggérant que les mêmes mécanismes expliquent la dégradation des résultats de ces deux catégories d'élèves.

Ces résultats suggèrent que d'autres facteurs que ceux liés aux conditions familiales de ces élèves interviennent dans le renforcement notable des inégalités de compétence entre natifs et non-natifs d'une part, et pour les élèves dont le capital culturel hérité est très faible d'autre part. Or, ces deux types d'élèves ont un point commun. Ils sont scolarisés dans les mêmes types d'établissements, ceux de la «périphérie» pour reprendre l'expression d'Agnès van Zanten (2012) ou encore ceux dont la ségrégation sociale se cumule avec la ségrégation ethnique et scolaire (Felouzis, 2003). Notre hypothèse est que ces transformations des inégalités relèvent d'inégales opportunités d'apprentissage car la nature de l'offre de formation proposée aux élèves en fonction de leur parcours migratoire et de leur capital culturel est elle-même inégale. Nous serions donc plus dans un schéma de discriminations systémiques envers les élèves non natifs qui se seraient accentuées en France au cours de la décennie 2000.

\section{Discussion : deux hypothèses explicatives}

Comment interpréter les évolutions de l'ampleur et de la structure des inégalités scolaires en France que nous mettons au jour dans cet article? La théorie de la discontinuité culturelle, si elle constitue un principe explicatif efficace pour comprendre une part des inégalités scolaires, n'est pas ici suffisante pour expliquer l'accroissement des inégalités de compétence entre élèves natifs et non natifs entre 2003 et 2012. Nous l'avons dit, l'explication la plus fréquente en France attribue les difficultés scolaires des élèves issus de l'immigration à leur appartenance massive aux classes sociales les plus défavorisées. Dans cette perspective, il suffirait que ces élèves voient leurs conditions sociales s'améliorer pour qu'ils rattrapent le niveau scolaire des élèves natifs. Or, nos résultats montrent que ce n'est pas le cas. On voit même qu'à statut socioéconomique équivalent, les élèves n'obtiennent pas les mêmes niveaux de compétence en fonction de leur statut migratoire. II nous faut donc comprendre la source de ce phénomène. Comment expliquer ce "plafond de verre» (glass ceiling) ${ }^{9}$ qui limite les acquis et les compétences des élèves non natifs, quel que soit leur capital culturel hérité?

À ce stade de notre raisonnement, deux hypothèses peuvent être mobilisées. La première hypothèse permettrait de «sauver » la théorie de la discontinuité culturelle en considérant que certaines informations non présentes dans nos sources empiriques PISA pourraient expliquer l'aggravation des écarts de score entre natifs et non-natifs. La France a en effet une conception très particulière des enquêtes internationales. Certaines variables, probablement considérées comme «sensibles» ou potentiellement «discriminatoires », ne sont pas renseignées. II s'agit notamment de la langue parlée (si la langue n'est pas le français, il est impossible de savoir de quelle langue il s'agit), du lieu de naissance des parents et des élèves de $1^{\text {re }}$ génération. La France est un des rares pays, parmi les 65 participants à l'enquête PISA, à masquer ces informations pourtant cruciales pour comprendre les phénomènes scolaires. Ainsi l'absence de ces données rend impossible de vérifier l'existence de différences, au sein même des élèves non natifs, liées aux origines nationales ou culturelles. Or, des travaux de recherche récents montrent que les élèves ont des comportements, des résultats scolaires, des rapports à l'école et des stratégies scolaires différenciés en fonction de leur origine nationale et culturelle et qu'ils font par ailleurs l'objet de traitements différenciés de la part de l'institution scolaire. Ainsi, le désavantage scolaire des élèves issus de l'immigration maghrébine est empiriquement renseigné grâce aux résultats de l'enquête Trajectoires et Origines (Brinbaum \& Kieffer, 2009; Brinbaum, Moguérou \& Primon, 2013). De même, Ichou (2013), reprenant à son compte les travaux de Sayad (1999), montre qu'une part non négligeable des inégalités liées aux parcours migratoires est attribuable à la situation socioéconomique des familles avant la migration, mettant ainsi en lumière la grande hétérogénéité que

9 La notion de plafond de verre dans le cas des carrières féminines est définie par Laufer (2005) comme «l'ensemble des obstacles visibles et invisibles qui séparent les femmes du sommet des hiérarchies professionnelles et organisationnelles» (p.31). 
recouvrent les notions d'élèves immigrés ou issus de I'immigration. Ainsi, si l'on suit cette hypothèse, l'accroissement des inégalités de compétence entre élèves natifs et non natifs pourrait être le fruit d'une transformation profonde des origines nationales et culturelles des élèves non natifs, accentuant ainsi la distance culturelle de ces élèves avec l'école, sans que I'on puisse la mesurer dans nos analyses.

Pour plausible qu'elle soit, cette hypothèse ne résiste pas longtemps à l'épreuve des faits. D'abord parce que les évolutions des vagues migratoires en France dans les années 2000 n'indiquent pas de changements assez profonds au plan des origines nationales et culturelles pour générer de telles transformations (INSEE, 2012). Ensuite parce que cette explication ne concernerait en toute logique que les élèves de $1^{\text {re }}$ génération, alors même que l'accroissement des inégalités concerne aussi les $2^{\mathrm{e}}$ génération. Enfin, parce qu'une telle explication ne pourrait rendre compte de transformations d'aussi grande ampleur que celles mises au jour par nos analyses.

La deuxième hypothèse, qui est pour nous plus pertinente et réaliste, est celle d'une discrimination systémique liée à la dégradation des conditions d'apprentissage dans les établissements qui scolarisent le plus d'élèves non natifs. Comment définir, de façon précise, ce que nous entendons par «discrimination systémique »? Pour Dhume (2011), celle-ci est difficile à mettre en évidence et elle est plus volontiers décrite comme "voilée» (van Zanten, 2006), ou indirecte. Selon Bataille et Schiff (1997), la discrimination systémique se distingue de la discrimination institutionnelle, elle est le résultat de l'ensemble des conduites des acteurs dont les effets convergent vers un phénomène discriminatoire. La discrimination systémique ne suppose donc pas de volonté explicite, volontaire et consciente de discriminer. Simon et Stavo-Debauge (2004) la définissent ainsi : "La discrimination systémique s'observe en dehors de l'intentionnalité et elle s'appréhende essentiellement dans les effets et conséquences d'un traitement. Celui-ci pourra être dit discriminatoire s'il affecte systématiquement, négativement et de façon disproportionnée, les personnes d'un groupe donné» (p.62). Or, les inégalités de conditions d'apprentissage et de la qualité de l'enseignement dispensé aux élèves de parcours migratoires et de milieux socioéconomiques différents ont été renseignées par des monographies d'établissements (Payet, 1995), par des analyses précises et informées des modes d'enseignement et des choix pédagogiques dans des contextes marqués par une forte ségrégation sociale et ethnique (van Zanten, 2012), ou encore par l'observation des pratiques d'enseignement dans divers contextes professionnels (Rochex \& Crinon, 2011). Que nous apprennent ces travaux? Que la composition sociale et migratoire des établissements constitue un puissant facteur de production des inégalités scolaires, que ce soit en France (Duru-Bellat, 2002; Felouzis, 2003) ou dans d'autres pays (Coleman, Campbell, Hobson et al., 1966; Hanushek \& Rivkin, 2006; Lauder, Thrupp, Goldstein et al., 2008; Van Ewijk \& Sleegers, 2010).

La ségrégation sociale et migratoire des établissements produit donc des inégalités par le biais des effets de composition en tant que tels, mais pas seulement. D'autres facteurs influent sur la qualité des établissements. Ces facteurs concernent l'ancienneté moyenne des enseignants (Hanushek \& Rivkin, 2006) dont on sait qu'elle est un facteur explicatif de leur efficacité (Bressoux, 1996). Ils concernent aussi le turnover des équipes et les pratiques pédagogiques des enseignants qui sont affectées par la nature du public auquel ils s'adressent (Paty, 1981; van Zanten, 2012). Dans cette perspective, ce serait la qualité différentielle des écoles qui expliquerait l'accroissement des inégalités de compétence et non la distance culturelle des élèves à l'école en elle-même. À l'appui de cette thèse, on peut citer l'augmentation notable de la part de variance des scores expliquée par l'établissement dans les enquêtes PISA (Felouzis \& Charmillot, 2012).

\section{Conclusion}

Nous avons montré que les inégalités de compétence entre élèves natifs et non natifs ont non seulement fortement augmenté dans la décennie 2000 en France, mais surtout que la structure de ces inégalités a changé. Notre thèse est qu'il s'agit d'un phénomène de discrimination systémique qui n'est au demeurant que partiellement opposée à la théorie de la discontinuité culturelle. Car l'éloignement des élèves non natifs à la norme scolaire ne doit pas être compris comme la cause de leur relatif échec scolaire, mais comme une conséquence des discriminations qu'ils subissent. Si I'on suit la démonstration de John Ogbu (1982), il est utile de distinguer plusieurs formes de discontinuité. La discontinuité universelle concerne l'ensemble des élèves dont les valeurs et pratiques familiales sont éloignées de celles promues par l'école. Ce type de dis- 
continuité peut notamment s'appliquer aux élèves de $1^{\text {re }}$ génération à faible capital culturel hérité, mais aussi aux natifs éloignés des normes scolaires par leur originale sociale. Toutefois, pour les $2^{\mathrm{e}}$ génération, que l'on pourrait comparer, mutatis mutandis, aux «minorités involontaires» d'Ogbu, il ne s'agit pas de ce type de discontinuité car elle ne permet pas de comprendre qu'une amélioration de leur capital culturel ne leur permette pas d'avoir les mêmes acquis que les natifs ${ }^{10}$. Il s'agit alors, toujours selon Ogbu, d'une discontinuité culturelle secondaire, résultat de la discrimination subie par ces populations. Dans le contexte nord-américain décrit par Ogbu, cette discrimination trouve son origine dans la barrière aux emplois que subit la minorité noire. Dans le cas français, on sait aussi qu'il existe des discriminations ethno-raciales sur le marché du travail (Safi, 2013). Celles-ci sont non institutionnalisées, mais n'en sont pas moins agissantes (Beauchemin, Hamel,

10 Il est à noter que, pour les élèves migrants ou issus de l'immigration, cette discontinuité universelle se situerait en amont de la migration (dispositions cognitives, conception du monde, etc.).

\section{Bibliographie}

BATAILLE P. \& SCHIFF C. (1997). «La discrimination à l'embauche. Le cas du bassin d'Alès». Les Annales de la recherche urbaine, «Ville, emploi, chômage», $\mathrm{n}^{\circ} 76, \mathrm{p}$. 89-96.

BEAUCHEMIN C., HAMEL C., LESNÉ M. \& SIMON P. (2010). "Les discriminations : une question de minorités visibles». Population et sociétés, $n^{\circ} 466$, p. 2-4.

BERNARD P.-Y. (2011). Le décrochage scolaire. Paris : PUF.

BOUDON R. (1973). L'inégalité des chances : la mobilité sociale dans les sociétés industrielles. Paris : Colin.

BOURDIEU P. (2013). Manet. Une révolution symbolique. Paris: Éd. du Seuil.

BOURDIEU P. \& PASSERON J.-C. (1964). Les Héritiers. Paris : Éd. de Minuit.

BOUVIER G. (2012). «Les descendants d'immigrés plus nombreux que les immigrés : une position française originale en Europe». Immigrés et descendants d'immigrés en France-INSEE Références, édition 2012. Paris : INSEE.

BRESSOUX P. (1996). «The Effects of Teachers' Training on Pupils' Achievement: The Case of Elementary Schools in France ». School Effectiveness and School Improvement: An International Journal of Research, Policy and Practice, $n^{\circ} 7(3)$, p. 252-279.

BRESSOUX P. (2008). Modélisation statistique appliquée aux sciences sociales. Bruxelles : De Boeck.
Lesné et al., 2010; Silberman \& Fournier, 2006). Les travaux d'Ogbu permettent donc de construire un lien entre discontinuité culturelle et discriminations systémiques et ainsi de prendre en compte le fait que d'autres facteurs que ceux liés aux conditions familiales interviennent pour expliquer l'accroissement des inégalités liées au statut migratoire et leur évolution en France dans les années 2000.

Georges Felouzis

Université de Genève, faculté de psychologie et des sciences de l'éducation, GGAPE Georges.Felouzis@unige.ch

Barbara Fouquet-Chauprade Université de Genève, faculté de psychologie et des sciences de l'éducation, GGAPE Barbara.Fouquet-Chauprade@unige.ch

Samuel Charmillot Université de Genève, faculté de psychologie et des sciences de l'éducation, GGAPE Samuel.Charmillot@unige.ch

BRINBAUM Y. \& KIEFFER A. (2009). "Les scolarités des enfants d'immigrés de la sixième au baccalauréat : différenciation et polarisation des parcours». Population, vol.64, n³, p.561-610.

BRINBAUM Y., MOGUÉROU L. \& PRIMON J.-L. (2013). «Trajectoires scolaires des descendants d'immigrés». In C. Bauchemin, C. Hamelle \& P. Simon (dir.), Trajectoires et Origines. Enquête sur la diversité des populations en France. Paris : INED.

BROCCOLICHI S., BEN AYED C. \& TRANCART D. (2010). École: les pièges de la concurrence. Comprendre le déclin de l'école française? Paris : La Découverte.

CLERC P. \& GIRARD A. (1964). « Nouvelles données sur l'orientation scolaire au moment de l'entrée en sixième ». Population, $\mathrm{n}^{\circ}$ 19(5), p. 829-872.

COLEMAN J. S., CAMPBELL E. Q., HOBSON C. F., MCPARTLAND J. M., MOOD A. M., YORK R. L. \& WEINFELD F. D. (1966). Equality of Educational Opportunity (U.S.G.P. Office, Trans.). Washington : U.S. Department of Health, Education and Welfare.

CRAHAY M. (2013). L'école peut-elle être juste et efficace? De l'égalité des chances à l'égalité des acquis. Bruxelles: De Boeck.

DHUME F. (2011). «La recherche française et la discrimination ethnico-raciale dans l'orientation scolaire : un problème 
de point de vue». Journal du droit des jeunes, n³08, p.28-32. En ligne: $<$ http://www.cairn.info/revue-journaldu-droit-des-jeunes-2011-8-page-28.htm > (consulté le 13 juillet 2015).

DUMAY X., DUPRIEZ V. \& MAROY C. (2010). «Ségrégation entre écoles, effets de la composition scolaire et inégalités de résultats ». Revue française de sociologie, n 51(3), p. 461-480.

DURU-BELLAT M. (2002). Les inégalités sociales à l'école. Genèse et mythes. Paris : PUF.

FELOUZIS G. (2003). «La ségrégation ethnique au collège et ses conséquences». Revue française de sociologie, vol. 44, n³, p.413-447.

FELOUZIS G. (2014). Les inégalités scolaires. Paris : PUF, coll. «Que-sais-je?».

FELOUZIS G. \& CHARMILLOT S. (2012). Les enquêtes PISA. Paris : PUF, coll. «Que sais-je?».

FELOUZIS G., CHARMILLOT S. \& FOUQUET-CHAUPRADE B. (2011). "Les inégalités scolaires en Suisse et leurs déclinaisons cantonales : l'apport de l'enquête PISA 2003 ». Revue suisse de sociologie, vol.37, n 1 , p. 33-55.

FELOUZIS G., CHARMILLOT S. \& FOUQUET-CHAUPRADE B. (2013). «Comment organiser l'enseignement secondaire obligatoire? Une politique publique et son analyse dans le canton de Genève ». Revue suisse de sociologie, n³9(2), p. $225-243$

FOUQUET-CHAUPRADE B. (2013). "L'ethnicité au collège : bien-être et effet de contexte». Sociologie, n 4(4), p. 431449.

GANZEBOOM H. B. G., DE GRAAF P. M. \& TREIMAN D. J. (1992), «A standard international socio-economic index of occupational status». Social Science Research, n²1(1), p. 1-56.

GRATIOT-ALPHANDÉRY H. \& LAMBIOTTE B. (1973). Le retard scolaire des enfants travailleurs migrants. Paris : Centre International de l'Enfance.

HALBWACHS M. ([1935] 1944). «La statistique en sociologie». In CIS (Centre international de synthèse), La statistique, ses applications, les problèmes qu'elle soulève. Paris : PUF, p. 113-134.

HANUSHEK E. A. \& RIVKIN S. G. (2006). «School Quality and the Black-White Achievement Gap». National Bureau of Economic Research Working Paper Series, nº 12651.

ICHOU M. (2013). «Différences d'origine et origine des différences : les résultats scolaires des enfants d'émigrés/ immigrés en France du début de l'école primaire à la fin du collège ». Revue française de sociologie, $n^{\circ}$ 54(1), p. 5-52.

INSEE (2012). Immigrés et descendants d'immigrés en France-INSEE Références, édition 2012. Paris : INSEE.

INSEE (2014a). Le recensement de la population. En ligne : <http://www.insee.fr/fr/> (consulté le 13 juillet 2015).

INSEE (2014b). «Les immigrés récemment arrivés en France. Une immigration de plus en plus européenne». INSEE Première, $\mathrm{n}^{\circ} 1524$.

LAHIRE B. (2008). La raison scolaire. École et pratiques d'écriture, entre savoir et pouvoir. Rennes: Presses universitaires de Rennes.
LAUDER H., THRUPP M., GOLDSTEIN H., ROBINSONT. \& KOUNALI D. (2008). Social Class, Pupil Composition, Pupil Progress and School Performance: An Analysis of Primary Schools. Working Paper. En ligne : <http://www.bath. ac.uk/research/harps/Resources/The\%20Effects\%20 of\%20Pupil\%20Composition\%20in\%20Primary\%20 Schools\%20wbl.pdf> (consulté le 13 juillet 2015).

LAUFER J. (2005). «La construction du plafond de verre : le cas des femmes cadres à potentiel ». Travail et emploi, $n^{\circ} 102$, p. 31-44.

MERLE P. (2012). «À qui profitent les dépenses éducatives?». La vie des idées. En ligne: $<$ http://www.laviedesidees.fr/Aqui-profitent-les-depenses.html> (consulté le 13 juillet 2015).

MERTON R. K. (1968). «The Matthew Effect in Science: The reward and communication systems of science are considered ». Science, $n^{\circ}$ 159(3810), p. 56-63.

OBERTI M. (2007). L'école dans la ville : ségrégation-mixitécarte scolaire. Paris : Presses de Sciences-Po.

OCDE (2005). PISA 2003 Technical Report. Paris : OCDE.

OCDE (2013a). Résultats de PISA 2012. Comment l'engagement des élèves, leur motivation et leur assurance peuvent les aider à apprendre. Paris : OCDE.

OCDE (2013b). Résultats du PISA 2012. Savoirs et savoir-faire des élèves : performance des élèves en mathématiques, en compréhension de l'écrit et en sciences. Paris : OCDE.

OGBU J. U. (1982). «Cultural Discontinuities and Schooling». Anthropology \& Education Quarterly, vol.13, n²4, p.290307.

PATY D. (1981). Douze collèges en France : le fonctionnement réel des collèges publics. Paris : La Documentation française.

PAYET J.-P. (1995). Collèges de banlieue. Ethnographie d'un monde scolaire. Paris : Méridiens Klincksieck.

ROCHER T. (2008). «Lire, écrire, compter. Les performances des élèves de CM2 à 20 ans d'intervalle 1987-2007». Note d'information de la DEPP, $\mathrm{n}^{\circ}$ 08-38.

ROCHERT. \& LE DONNÉ N. (2012). «Les difficultés de lecture en début de sixième. Évolution à dix ans d'intervalle 19972007». Éducation et formations, n82, p.30-37.

ROCHEX J.-Y. \& CRINON J. (2011). La construction des inégalités scolaires. Au cœur des pratiques et des dispositifs d'enseignement. Rennes: Presses universitaires de Rennes.

SAFI M. (2013). Les inégalités ethno-raciales. Paris : La Découverte.

SAYAD A. (1999). La double absence. Des illusions de l'émigré aux souffrances de l'immigré. Paris : Éd. du Seuil.

SILBERMAN R. \& FOURNIER I. (2006). «Les secondes générations sur le marché du travail en France : une pénalité ethnique ancrée dans le temps. Contribution à la théorie de l'assimilation segmentée ». Revue française de sociologie, vol.47, n², p. 243-292.

SIMON P. \& STAVO-DEBAUGE J. (2004). «Les politiques antidiscriminations et les statistiques : paramètres d'une incohérence ». Sociétés contemporaines, n54, p.57-84.

SUCHAUT B. (2015). «Les inégalités sociales de réussite à 
l'aune de la mesure des performances des élèves. Le cas vaudois ». In G. Felouzis \& G. Goastelec, Les inégalités scolaires en Suisse. École, société et politiques éducatives. Berne : Peter Lang, p.67-94.

UNESCO (2006). International Standard Classification of Education, ISCED 97. Montréal : Unesco institute for statistics.

VALLET L.-A. (2002). Sur l'analyse de régression en sociologie. Communication présentée aux 39e Journées de Statistique, organisées par la Société française de statistique à Angers du 11 au 15 juin 2007. En ligne: <http://www. sfds.asso.fr/ressource.php?fct=ddoc\&i=834> (consulté le 13 juillet 2015).

VALLET L.-A. \& CAILLE J.-P. (1996). «Les élèves étrangers ou issus de l'immigration dans l'école et le collège français. Une étude d'ensemble». Les Dossiers d'éducation et formations, $\mathrm{n}^{\circ} 67$.
VAN EWIJK R. \& SLEEGERS P. (2010). «The effect of peer socioeconomic status on student achievement: A metaanalysis». Educational Research Review, n 5(2), p. 134-150.

van ZANTEN A. (2006). «Une discrimination banalisée? L'évitement de la mixité sociale et raciale dans les établissements scolaires ». In D. Fassin \& E. Fassin (dir.), De la question sociale à la question raciale? Représenter la société française. Paris : La Découverte, p. 195-210.

van ZANTEN A. (2012). L'école de la périphérie. Scolarité et ségrégation en banlieue. Paris : PUF.

van ZANTEN A. \& ANDERSON-LEVITT K. (1992). «L'anthropologie de l'éducation aux États-Unis : méthodes, théories et applications d'une discipline en évolution». Revue française de pédagogie, n¹01, p.79-104.

WILLIS P. ([1978] 2011). L'école des ouvriers. Paris : Agone. 


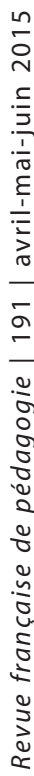

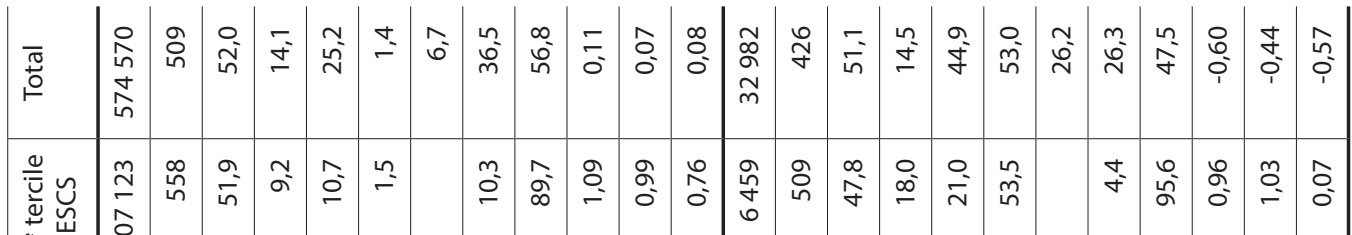
竞

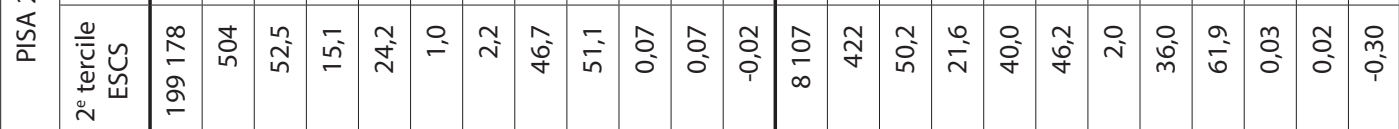

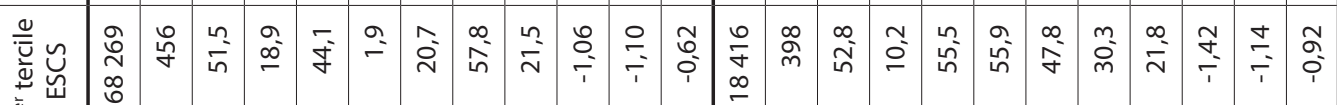
○े

«

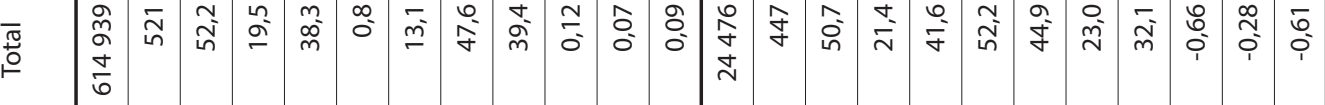

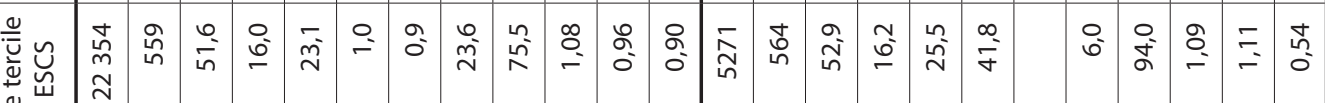
i

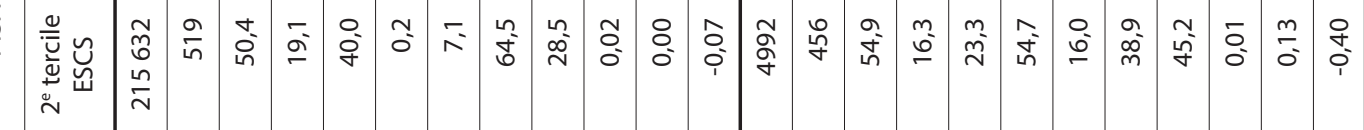

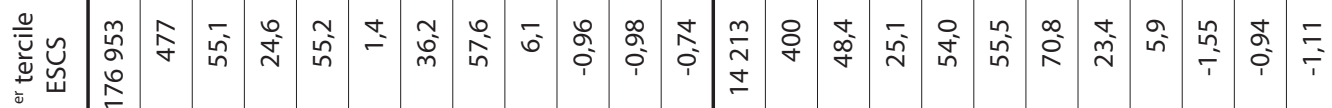

si

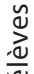

$\frac{\tilde{g}}{0}$

$\frac{\tilde{v}}{\sigma}$

$\frac{\sqrt{2}}{\frac{\pi}{2}}$

$\stackrel{\frac{\pi}{0}}{\frac{\pi}{6}}$

$\frac{\tilde{g}}{0}$

을

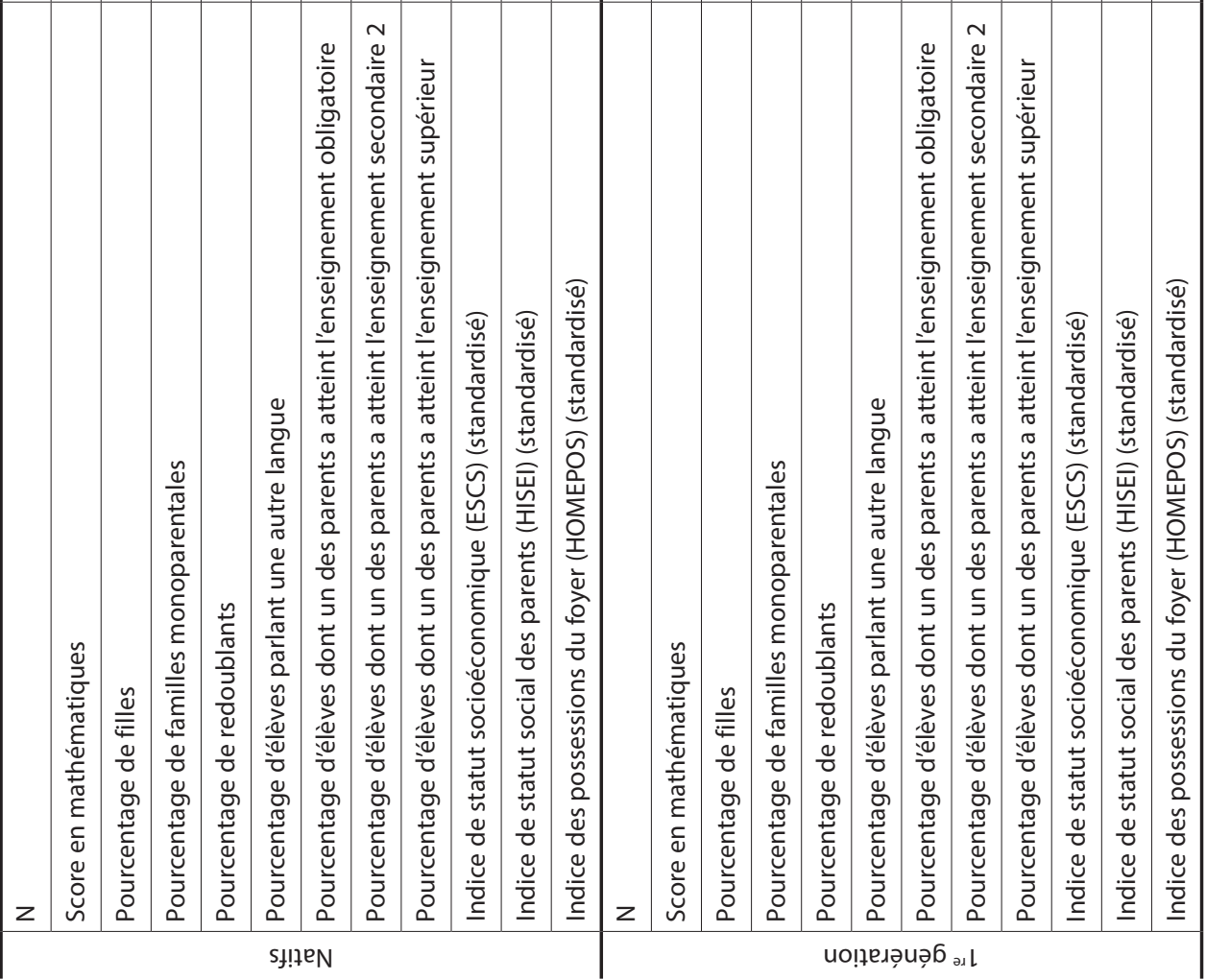




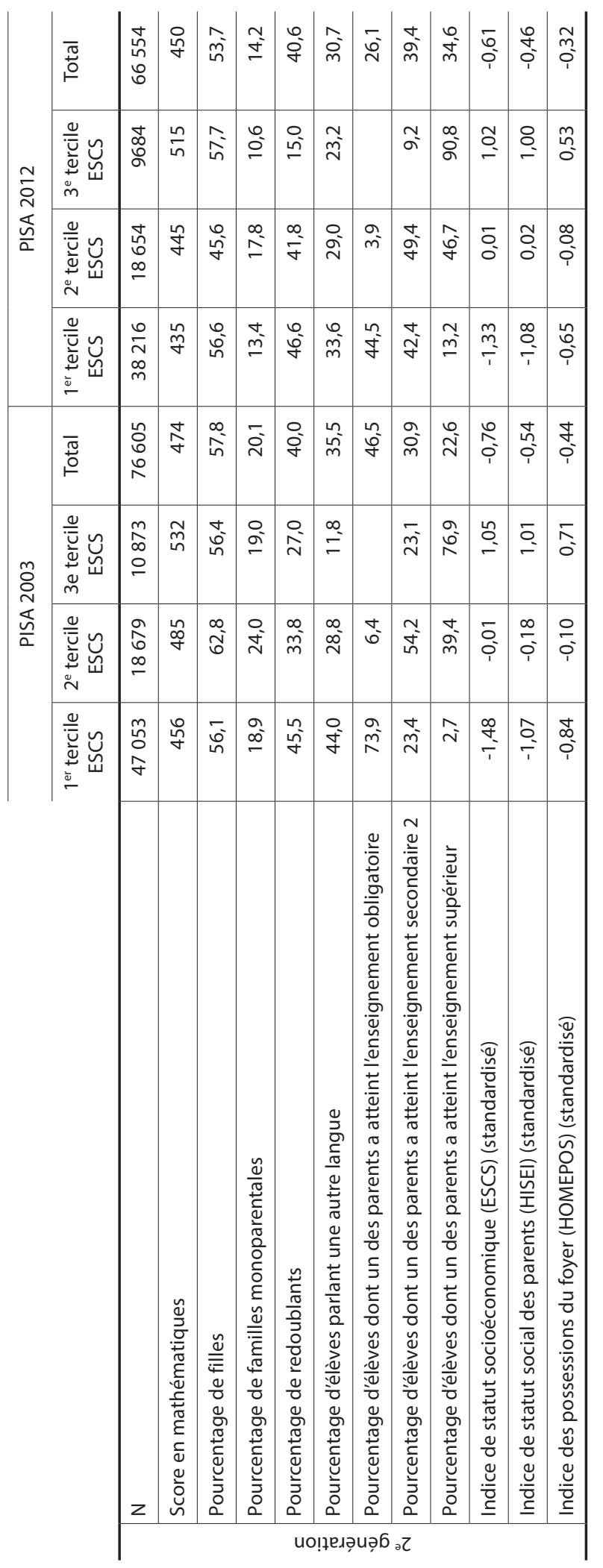

